\title{
Resiliencia empresarial: análisis empírico de Aguas de Cartagena S.A. E.S.P.
}

\author{
Business resilience: empirical analysis of Aguas de Cartagena S.A. E.S.P.
}

Javier A. Mendoza-Betin

Aguas de Cartagena S.A - Colombia

ORCID iD: https://orcid.org/0000-0002-8355-8581

jmendoza@acuacar.com
Fecha de recepción: 02/11/2020

Fecha de evaluación: 10/02/2021

Fecha de aceptación: 20/04/2021

Cómo citar: Mendoza-Betin, J. (2021). Resiliencia Empresarial: análisis empírico de aguas de Cartagena S.A. E.S.P. Revista Cientifica Anfibios, 4(1). https://doi.org/10.37979/afb.2021v4n1.80

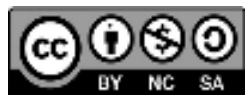

Atribución-NoComercial-CompartirIgual 4.0 Internacional

\section{Resumen}

El articulo describe los resultados del ejercicio ad hoc de resiliencia realizado por Acuacar de cara al Covid-19. La investigación por una parte responde la pregunta central en torno a la capacidad de recuperación de las operaciones suspendidas a raíz de la emergencia sanitaria. El diseño metodológico para esto está soportado en la técnica de Design Thinking lo que posibilitó 112 propuestas de mejora. Por otra parte, se contrastó la hipotesis de que la creatividad, la integración digital, el trabajo en equipo y el análisis de la información mejoran la resiliencia. Se concluye entonces que la gestión de resiliencia implica el involucramiento holístico tanto de sus rutinas y acciones como de sus colaboradores. En síntesis, se corrobora la tesis sobre que la gestión de resiliencia necesita el implicamiento integral y consciente tanto de sus procesos como de los empleados a diferencia de solo la implementación de acciones antropomorfizadas así como también se requiere de creatividad, recaudo, análisis y evaluación de la información y trabajo en equipo.

\section{Palabras Clave}

Resiliencia empresarial; procesos; colaboradores

\begin{abstract}
The article describes the results of the ad hoc resilience exercise carried out by Acuacar in the face of Covid- 19 . The investigation, on the one hand, answers the central question regarding the recovery capacity of the operations suspended as a result of the health emergency. The methodological design for this is supported by the Design Thinking technique, which enabled 112 proposals for improvement. On the other hand, the hypothesis that creativity, digital integration, teamwork and the analysis of information improve resilience was contrasted. It is therefore concluded that resilience management implies the holistic involvement of both its routines and actions and its collaborators. In summary, the thesis is corroborated that resilience management needs the comprehensive and conscious involvement of both its processes and employees, as opposed to only the implementation of anthropomorphized actions as well as creativity, collection, analysis and evaluation of information and teamwork.
\end{abstract}




\section{Keywords:}

Business resilience; processes; collaborators

\section{Introducción}

El mercado y las empresas en la actualidad son cada vez más complejas dadas situaciones especialmente externas, pero ha surgido la resiliencia corporativa como una acción holística y sistémica que ha posibilitado reconfigurar las operaciones de las firmas garantizando su continuidad. Planear y organizar a condición de la resiliencia es en este momento el mayor reto que necesitan resolver las compañías como un medio para mejorar la preparación para evitar y mitigar las amenazas, ya sean naturales o provocadas por el hombre. La complejidad en consecuencia se ha abordado de manera frecuente como impredecible, inexplicable, indeterminada y una condición suficientemente peligrosa debido a las incertidumbres relacionadas con el ambiente de las amenazas e indecisiones relacionadas con la acción de coordinación en respuesta a ellas.

Al configurar los sistemas de respuesta (e.g. gestión de crisis, de desastres, de seguridad, protección, planes de resiliencia y continuidad del negocio), se requieren enfoques organizacionales integrales, de múltiples niveles y perspectivas que, involucren no solamente los procesos y operaciones de las empresas sino sus colaboradores. Por lo tanto, la resiliencia ha llamado la atención de los responsables políticos, profesionales y académicos, quienes preocupados por identificar niveles suficientes de recuperación han posibilitado construir su literatura teórica y práctica al menos en los últimos 20 años; empero, no ha tenido tal importancia en el contexto colombiano, de hecho, existen muy pocos trabajos documentados en torno a dicha fenomenología. La resiliencia en tal virtud como una cualidad adaptativa de las personas y las organizaciones que lo implementan es el tema central del presente artículo.

El trabajo de investigación posibilitó admitir la posición de la resiliencia empresarial holística respaldada en las tesis de Hamel y Välikangas (2003), Hoffman (2014), McManus, Seville, Brunsdon y Vargo (2007) y Prezelj y Doerfel (2017), pero contraviniendo el punto de vista de Meneghel, Salanova y Martínez
(2013), Salanova, Llorens, Cifre y Martínez (2012) y Somsing y Belbaly (2017), quienes las consideran antropomorfizadas e individuales y grupales. El método de design thinking propició fortalecer la capacidad de recuperación de las operaciones realizadas con contratistas de la empresa Aguas de Cartagena S.A. E.S.P. de cara al Covid-19 porque del $50 \%$ de los procesos suspendidos a raíz de la emergencia sanitaria en la actualidad solo continúan paralizados el 3\%, lo anterior en respuesta a las 112 de 223 propuestas entregadas por los equipos de trabajo que conformaron el ejercicio práctico. Por su parte, en el paradigma positivista, los factores: creatividad, integración digital, trabajo en equipo y análisis de la información numérica afectaron positivamente la resiliencia empresarial.

\section{Fundamentación teórica}

La resiliencia organizacional usualmente se examina dentro del marco de una rutina dinámica con fundamento en sus numerosas definiciones. La primera aborda la perspectiva de problemas, es decir, saber y poder sortear los eventos inesperados y recuperarse de ellos rápidamente. Desde otra corriente, se analiza a partir de la necesidad de captar alertas tempranas del mercado y señales débiles corporativas a condición de eficientarlas. En el tercer enfoque, es imprescindible aprender de los eventos pasados, estudiar los conflictos e intercambiar información entre actores externos e internos, en orden de optimizar las debilidades institucionales y capacitar los gerentes. No en vano, la resiliencia corporativa generalmente se estudia en consecuencia, como un proceso resolutivo continuo que, requiere atención iterativa multi e interdisciplinaria.

Conceptualmente, la resiliencia empresarial se ha discutido principal e internacionalmente y con pocos trabajos en el ámbito colombiano como una función gerencial de los ecosistemas organizacionales participativos e interdependientes lo que implica la inflexión de (i) aptitudes y métodos para ser flexibles y adecuarse fácilmente a nuevos contextos inesperados en el corto y mediano plazo, (ii) actividades y ap- 
titudes para reconocer fructuosa y rápidamente cambios de improviso en el corto y mediano plazo, (iii) habilidades y rutinas para recuperarse de acontecimientos adversos en el corto y mediano plazo, (iv) prácticas y experiencias para conservar las funciones y los resultados en medio de la tensión en el mediano plazo y (v) gestión creativa de los riesgos, crisis y continuidad de los negocios (Garcia-Merino et al., 2015; Mendoza, 2013; Mendoza-Betin, 2019; Prezelj y Doerfel, 2017 y Somsing y Belbaly, 2017).

Entre las constricciones y limitaciones a la resiliencia corporativa se incluyen los eventos adversos naturales, las sustancias peligrosas, los ataques cibernéticos, los actos terroristas y las enfermedades infecciosas que podrían afectar las comunidades y los negocios. El cuerpo literario de los últimos años incluye gran diversidad de trabajos teóricos y empíricos, desde escrutinios técnicos hasta investigaciones científicas sociales y clínicas, los que abordan principalmente la prevención y la planificación de las situaciones mencionadas. De hecho, el liderazgo, la dirección de los equipos gerenciales y las conexiones organizacionales también fueron puestas a prueba en la resiliencia organizacional, particularmente en los momentos de crisis, pero no en detalle como sucede con la pandemia de la Covid-19 en el contexto empresarial colombiano, de facto, a la fecha no se conocen exámenes prácticos al respecto en la ciudad de Cartagena. Inmediatamente la revisión teórica y empírica pertinente en los entornos internacional y nacional permitió la construcción aproximada del estado del arte de la presente investigación:

Con base en los trabajos más importantes en torno a la resiliencia en negocios, el investigador propone tres categorías de clasificación: La primera se denomina resiliencia empresarial holística donde se encuentran puntos de convergencia alrededor de que las organizaciones deberán prepararse para enfrentar lo inesperado a través del pensamiento de sistemas sociales y la activación de la participación de sus colaboradores. En esta perspectiva, aparece Sutcliffe y Vogus (2007), los que descubrieron que resulta inevitable para la seguridad de las corporaciones que estas dependan de comportamientos que, entre otros componentes, analicen la cultura de seguridad y mitigación de los riesgos en los procesos, el liderazgo y la dirección de la continuidad del negocio, la coordinación de la estrategia y las operaciones al igual que el compromiso y la confianza de sus colaboradores, lo que se estudió con fundamento en el contraste de contenido teóricos y observaciones empíricas en una muestra de hospitales.

Por su parte, McManus, Seville, Brunsdon y Vargo (2007) sustentan que la gerencia de la resiliencia corporativa envuelve 5 factores de la mayor importancia: (1) crear conciencia sobre los problemas de resiliencia por medio de planes permanentes de continuidad del negocio a manera de prácticas recurrentes, (2) Seleccionar los componentes organizativos esenciales para diseñar y actualizar los planes acotados, (3) autoevaluar conscientemente la vulnerabilidad de las operaciones, (4) identificar y priorizar las debilidades clave y (5) aumentar la capacidad de adaptación de los empleados. Estos elementos resultaron de una investigación realizada en 10 organizaciones en Nueva Zelanda. De manera parecida, Minolli (2005), consiente que, las empresas resilientes demandarán más que procesos; lideres creativos e imaginativos; estructuras, operaciones y organización del trabajo flexibles; y estrategias y cultura organizacional hincada sobre la base de soluciones inteligentes y participativas de los problemas, por lo que es necesario incluirlo en esta perspectiva.

Dentro de esta vertiente, Hamel y Välikangas (2003), argumentan que la esencia de la resiliencia corporativa reposa en su capacidad de generar opciones estratégicas y realinear sus recursos más rápido que sus rivales lo que generará sin lugar a duda ventajas estratégicas decisivas sobre las turbulencias del mercado. Del mismo modo cognitivo, Medina-Salgado (2012) admiten que, las empresas resilientes enseñan una cultura afín a capacidades de adaptación, agilidad e innovación, lo cual se basa en los siguientes componentes: (i) orientación permanente hacia la resistencia y mejora permanente, (2) comprensión de la situación; amenazas y oportunidades accionadas por el negocio por medio del seguimiento activo de las señales del mercado y (3) gestión de riesgos y crisis. Consonante a esta óptica, 
Mendoza-Betin (2018) sostiene que, la capacidad dinámica de resiliencia empresarial influye positivamente sobre la rentabilidad financiera en razón a que competencias (habilidades humanas) y procesos (actividades organizacionales) trabajando de la mano, mejoran su capacidad de ser flexible y adaptarse continuamente a nuevas situaciones imprevistas en el corto y mediano plazo.

Añádase a esta corriente la visión de Prezelj (2014), quien defiende la tesis de que una estrategia exitosa contra constricciones empresariales, en especial, el terrorismo y las guerras virales, deberán proporcionar capacidades para la cooperación interorganizacional y su red horizontal de abastecimiento, al igual que, la capacidad de manejar muchos desafíos interorganizacionales conexos. En síntesis, asegura, las corporaciones deberán adoptar un enfoque de red integral para las amenazas. Dentro de este marco ha de considerarse también el punto de vista de Hoffman (2014), quien hace referencia a gestionar efectivamente las crisis, es decir, sortear las situaciones difíciles que podrían producir resultados inesperados para las empresas. Este paradigma en consecuencia es susceptible también de convertirse en realidades encomiables las que podrían transformar una oportunidad en hitos de cambios e innovación, para de esta forma, determinar factores para salir adelante y sobretodo fortalecidos.

Es interesante examinar el presente planteamiento ahora desde la mirada de Doerfel (2016), quien concibe la organización como una red de procesos de comunicación que se complementan de las formas jerárquicas de poder y de control, pero las dos formas de comunicación, en red y jerárquica, coexisten. Estas rutinas sientan las bases para la resiliencia empresarial como un amortiguador anticipado de los problemas e imprevistos lo que, en ultimas, les permitirá a las compañías recuperarse rápidamente de las crisis. Es significativa la importancia que tienen Prezelj y Doerfel (2017) dentro de esta postura porque arguyen que, la resiliencia organizacional es la habilidad para contestar o ripostar al ambiente corporativo en medio de condiciones difíciles que pudieran situarlas en riesgo, se trata entonces de una pericia análoga a las capacidades de atención del entorno y aprovechamiento de oportunidades (o de ejecución). La conjunción de estas aptitudes de estoicismo y ejecución, junto con la adecuada gestión de riesgos y crisis, fue la que motivó a Mendoza (2013) a añadir una nueva expresión al corpus hipotético de las capacidades dinámicas, a la que denominó: capacidad de bisiliencia corporativa, que, incluye los componentes antes anotados.

Finalmente, Mendoza-Betin (2019) destaca la correspondencia que debe concurrir entre los distintos mecanismos culturales, estructurales, operativos y diferenciados a nivel corporativo, en orden de conformar y desplegar su resistencia, destacándose en ellos: la estructura organizativa, el liderazgo y el estilo de mandato, los procesos decisorios, el pensamiento gerencial, la cultura financiera y el diseño de nuevos procesos, productos y servicios. En similares términos se expresan Garcia-Merino, et al., (2015) al señalar que para poder crear y fructificar la resiliencia es indispensable que la organización conjugue dos ingredientes claves: acumulación de aprendizajes, por un lado, y generación de conocimientos y experiencias, por el otro; pudiendo ser esta la razón por la cual dos años más tarde, tanto Prezelj \& Doerfel (2017), realzaron la misión creativa de los riesgos y los conflictos en la resiliencia, asemejándola a la base que sustenta la continuidad de la firma en su sector.

El segundo grupo taxonómico se designa bajo el nombre de resiliencia antropomorfizada donde la presencia del intelecto y la experiencia de los seres humanos en la resiliencia empresarial impera por encima de la mística y orden corporativo. Al respecto conviene decir que Somsing y Belbaly (2017) insisten que la creatividad se convierte es una de las habilidades clave para los gerentes en la toma de decisiones estratégicas sobre todo en lo que concierne a la mitigación de riesgos. Podrían considerarse en consecuencia como ineludibles las siguientes rutinas gerenciales: (1) habilidades que permitan generar diferentes niveles de creatividad, y (2) un proceso dinámico que evoluciona y se afina con el tiempo. Dentro de este contexto cabe el paradigma de Salanova, Llorens, Cifre y Martínez (2012) los que realizaron dos estudios bajo posición positivista para validar el denominado Modelo de Organización Saludable y Resiliente (HERO por su 
sigla en inglés), donde demostraron que, la excelencia operacional y funcional de los empleados predice positivamente la lealtad del cliente y la satisfacción con la empresa en términos de resiliencia en situaciones coyunturalmente adversas.

Dentro de este marco epistemológico ha de considerarse dado su relevancia el enfoque de Hollnagel (2006), quien introduce el termino de Ingeniería de la Resiliencia (Resilience Engineering por su nombre en inglés) para referirse a la importancia de aprender sobre el lado positivo de la gestión de la seguridad en cualquier sector económico y corporativo hincándose en la adaptación y recuperación de las adversidades con base en el personal de primera línea quienes en conjunto con el equipo gerencial estudiarán de las situaciones adversas para terminar fortalecidos centrándose en la interrelación proactiva de mitigación de las crisis y riesgos sobre la base del conocimiento de las organizaciones como sistemas dinámicos.

Es interesante examinar el constructo también desde el planteamiento llamado resiliencia en las compañias a partir de lo individual $y$ grupal. Es significativo la importancia que tiene la posición de Luthar, Cicchetti y Becker (2000), quienes sostienen que, la resiliencia empresarial radica en los individuos que conforman las organizaciones principalmente en los miembros de los grupos gerenciales que serán los responsables de imprimir la capacidad de recuperar la organización de los constantes cambios que sufre, al margen de determinar y evaluar las condiciones usualmente inestables de sus ecosistemas para transfórmalas en mejoramiento continuo. Esta consideración es complementada por la propuesta de Meneghel, Salanova y Martínez (2013) los que establecen que la resiliencia empresarial comienza por la individual, es decir, por la capacidad emotiva y de desempeño de los colaboradores frente a situaciones adversas, lo cual necesita tener entonces colaboradores motivados y psicológicamente sanos al igual que proporcionar al equipo de las capacidades para afrontar los fracasos, los retrasos, los conflictos o cualquier otra amenaza de su bienestar grupal.

Finalmente, al interior de la categoría acotada surge la posición de (Arraut, comunicación personal, 23 de agosto de 2020) quien desde su experiencia como consultor en emprendimiento empresarial y Director de la Maestría de Innovación de la Universidad Tecnológica de Bolivar resalta que, la resiliencia empresarial está íntimamente asociada a los siguientes factores: (1) creatividad, (2) recopilación y evaluación de la información, (3) trabajo en equipo y (4) análisis de la información numérica. Con base en su experiencia, se diseñó un cuestionario ad hoc utilizando una escala Likert de 5 puntos, que va desde ( 1 =fuertemente en desacuerdo) a ( $5=$ totalmente de acuerdo) en orden de valorar la confiabilidad de los ítems. Dicha herramienta fue sometida a juicio de tres expertos y una prueba de atestamiento no probabilística de 15 respondientes a condición de validar los constructos y las escalas de medida, todos funcionarios de Acuacar, lo cual se ampliará en los apartados siguientes.

Ante el vacío de conocimiento en relación con trabajos asociados a la resiliencia empresarial en la ciudad de Cartagena (Colombia) y los pocos realizados en otras regiones de este país, surge la oportunidad de desarrollar una investigación que posibilite contribuir con sus hallazgos a la Teoría de la resiliencia, partiendo de una empresa importante en dicha ciudad. Por lo tanto, se considera acertado materializar el objetivo de la investigación: fortalecer la capacidad de resiliencia operativa en la empresa Aguas de Cartagena S.A. E.S.P. "Acuacar" para volver a operar los procesos suspendidos en medio del Covid-19. Por último, en orden mas no en trascendencia y a manera de cierre, se tiene que las organizaciones tendrán que prepararse sistemáticamente en respuesta a lo inesperado por medio de ejercicios de continuidad del negocio en el marco de planes de resiliencia a condición de mitigar sus fallas, riesgos, desviaciones, señales débiles en lo estratégico y operativo en conexidad con colaboradores para desempeñarse mejor. De todo lo anterior, surge la pregunta: ¿cómo robustecer la resiliencia organizacional en la empresa Aguas de Cartagena de tal suerte que le permita operar normalmente en tiempos Covid-19? Es de aclarar que cerca del $50 \%$ de las operaciones de cara al cliente en la empresa realizadas por contratistas fueron suspendidas a causa de la declaración de emergencia sanitaria del gobierno colombiano en el mes de marzo de 2020. 
Sin embargo, ante la posibilidad de complementar el estudio con un ejercicio numérico en Acuacar emergen las siguientes conjeturas, basadas en las variables conceptuales de (Arraut, comunicación personal, 28 de agosto de 2020) y Somsing y Belbaly (2017):

Hipótesis H1: La creatividad mejora la resiliencia empresarial.

Hipótesis H2: La recopilación y evaluación de la información mejora la resiliencia.

Hipótesis H3: El trabajo en equipo mejora la resiliencia empresarial.

Hipótesis H4: El análisis de la información numérica mejora la resiliencia.

\section{Método}

El análisis empírico fue diseñado primeramente bajo un método exploratorio ad hoc de corte longitudinal por espacio de 4 meses (inició en abril y finalizó en julio de 2020), soportado en la técnica de Design Thinking con lo cual se trazó la estrategia para restablecer el $50 \%$ de las operaciones ejecutadas por provee- dores de servicios de Aguas de Cartagena S.A. suspendidas a raíz de la declaratoria de emergencia sanitaria a causa del Covid-19 en el mes de marzo de 2020 el que se complementó con un análisis positivista. A continuación, las etapas para el primer examen, las que fueron diseñadas y ejecutadas de acuerdo con su necesidad y ocurrencia, empero; a su vez entregó datos e información pertinente, la que fue preciso problematizar (definir), proponer (idear) y viabilizar (prototipar) mediante la técnica señalada.

Etapa 1. Conocimiento del impacto de la crisis sanitaria Covid-19 para problematizar o definir como se le conoce a este período en Design Thinking.

- Análisis del impacto frente a la operación y el servicio de Acuacar.

- Examen de las consecuencias de cara a la continuidad del negocio desde los ámbitos económico, legal, social y reputacional.

- Definición de líneas estratégicas impactadas por la crisis sanitaria.

En seguida las líneas estratégicas definidas para adelantar el estudio:

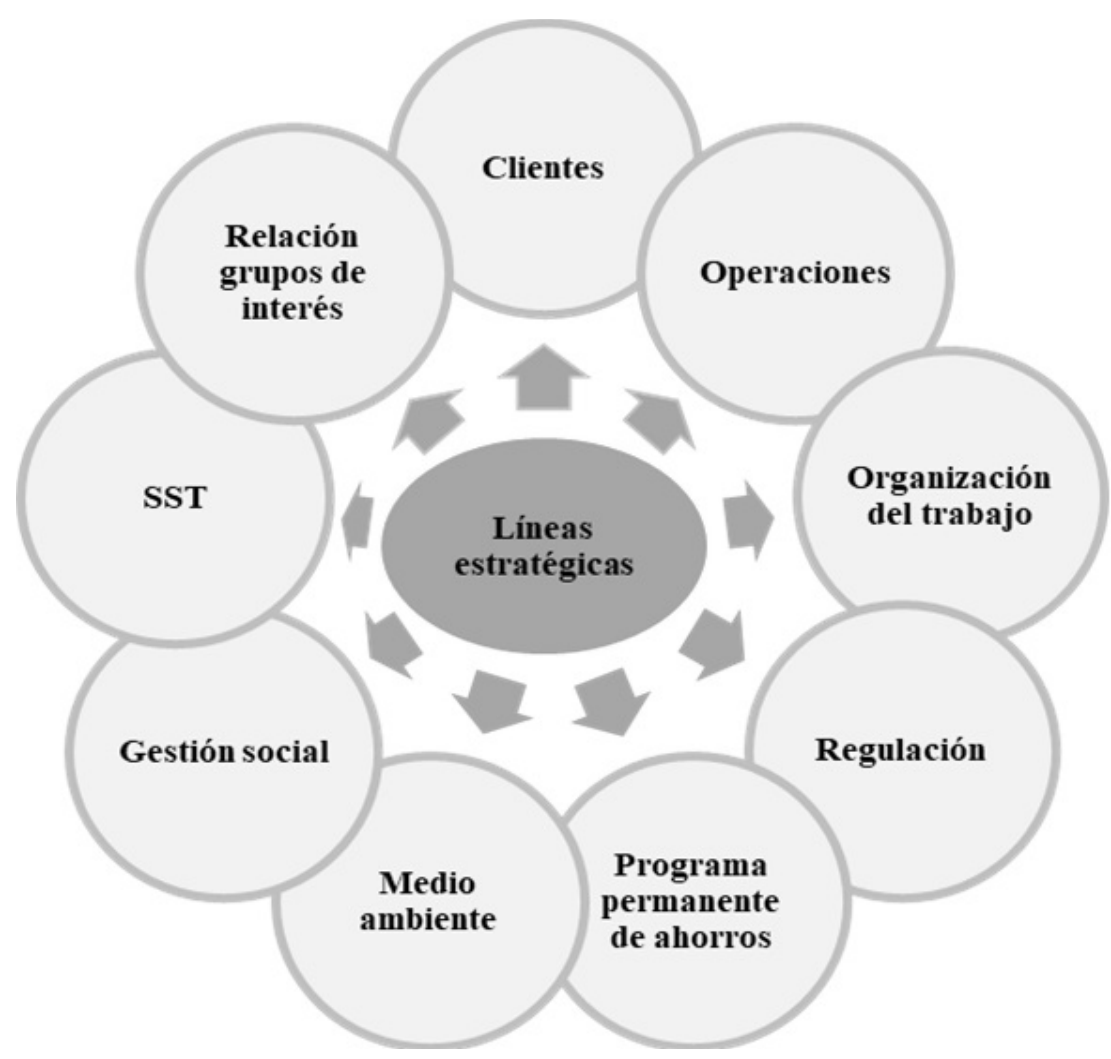

Figura 1. Líneas estratégicas definidas por el Comité de Resiliencia Fuente: Tomado de Acuacar (2020) 
Etapa 2. Análisis de medidas adoptadas para operar en situación de contingencia por asunto clave o línea estratégica del negocio a condición de proponer ideas que eliminen o reduzcan los problemas, es decir, su suspensión.

- Principales limitaciones o barreras para responder a requerimientos de la operación y los grupos de interés.

- Identificación de oportunidades / Lecciones aprendidas.
- Definición de grupos de interés. Se precisaron los siguientes: Clientes, accionistas, colaboradores, proveedores, contratistas, socio operador, aliados y autoridades, comunidades vulnerables, medios de comunicación y gremios.

- Coordinación de equipos de trabajo. En la tabla 1, las unidades de trabajo.

Tabla 1. Equipos de trabajo y sus funciones

Equipos de trabajo Funciones

1. Facilitadores o influencia- Responsables de articular las propuestas estratégicas y tácticas de dores los grupos focales a las operaciones, procedimientos, instrucciones de trabajo y niveles jerárquicos de mandos medios e inferiores de la compañía.

2. Grupos focales por línea es- Construyeron las propuestas estratégicas y tácticas por línea estratratégica que tiene a cargo tégica.

un líder.

Fueron liderados por los miembros del Comité de Resiliencia.

Se apoyaron en los grupos de facilitadores o influenciadores.

Pudieron invitar expertos externos acorde a temática o línea estratégica.

Presentaron las propuestas de conformidad al esquema diagramático de la Figura 3.

3. Comité de Resiliencia Consolidaron y analizaron las propuestas derivadas de grupos focales.

Validaron las propuestas en el Comité de Dirección en su papel de pares o expertos, en total hubo 12 profesionales.

Se reunieron diariamente de 5 a $6 \mathrm{pm}$ a revisar las propuestas.

4. Comité de Dirección o CO- Tomaron decisiones frente a las propuestas presentadas por miemDIR bros del Comité de Resiliencia.

Suministraron lineamientos para su adopción o implementación.

Se reunieron semanalmente.

Fuente: Tomado de Acuacar (2020)

Etapa 3. Recuperación y retorno a la nueva realidad

- Determinación de procesos que continuaron operando como antes de iniciar la crisis sanitaria (No son modificables).
- Definición de operaciones que fueron susceptibles de modificarse u optimizarse, a partir de las lecciones aprendidas.

- Propuestas de operaciones o procesos ajustados o modificados.

- Estrategias de relacionamiento con grupos de interés, acorde a la nueva realidad. 

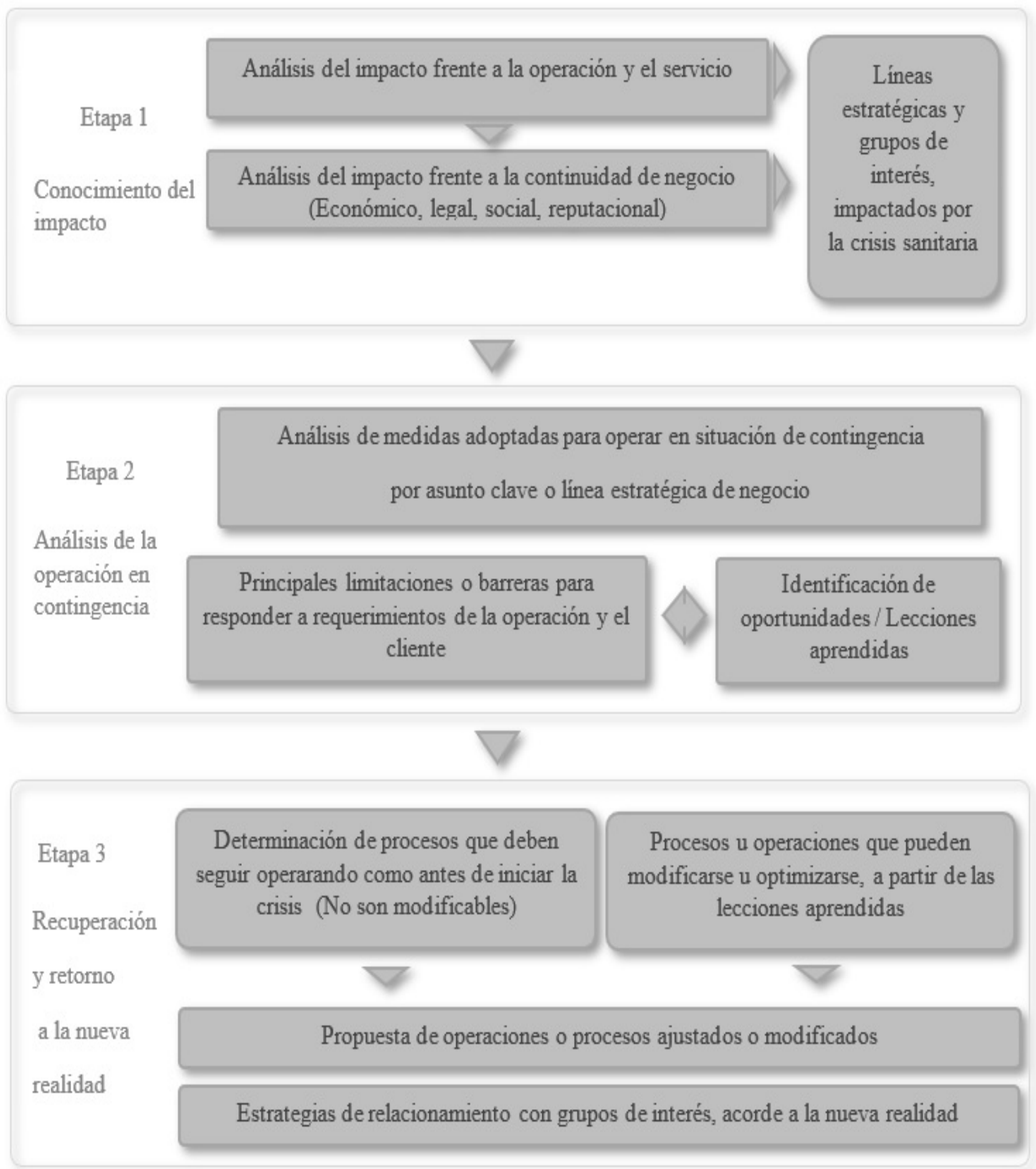

Figura 2. Etapas de la metodología del trabajo

Fuente: Tomado de Acuacar (2020)

Durante las etapas aludidas se presentaron propuestas (para restablecer las operaciones suspendidas a causa del Covid-19) para su validación por parte de los líderes de los grupos focales al Comité de Resiliencia y éste, al Comité Directivo. Las ideas resultaron de la aplicación de un instrumento de recogida de información sometido a doble proceso de validación: 1 . mediante juicio de 4 expertos; y 2 . a través de una prueba piloto en la que participó una muestra deliberada de 12 miembros del Comité de Resiliencia de ACUACAR. Después del estudio piloto, se aplicó dicho mecanismo a los grupos focales en tres rondas detalladas en la Figura 3. 


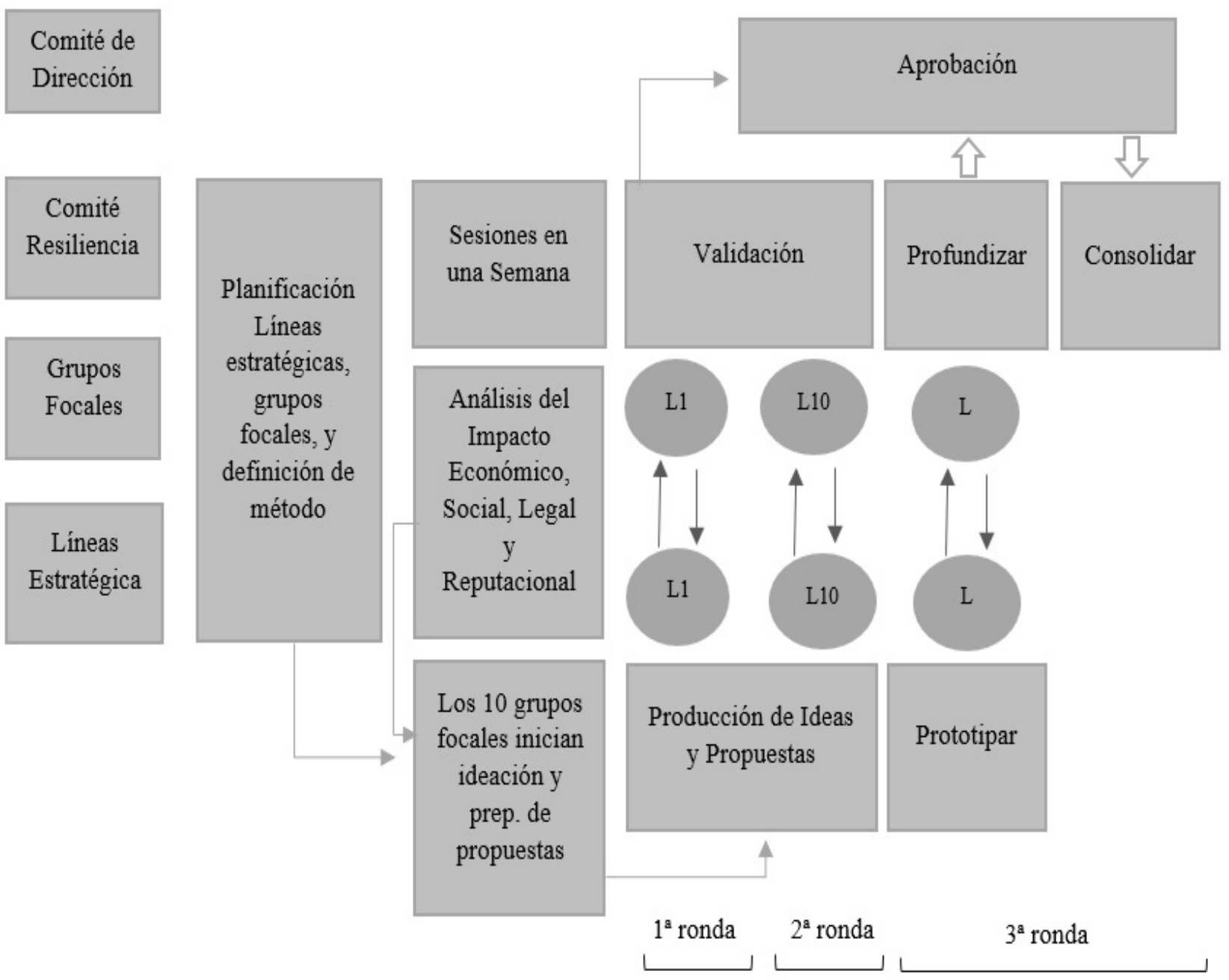

Figura 3. Esquema diagramático de la validación de las propuestas Comités de Resiliencia y Directivo Fuente: Tomado de Acuacar (2020)

En la Figura 4, se exponen las etapas por las sign Thinking. En la tercera ronda, se viabilizarán que pasaron las propuestas en la primera y segun- las iniciativas capaces de prototiparse, es decir, da ronda de validación de los Comité de Resilien- las que circunscriben exámenes experimentales. cia y Directivo, donde se aplicó la técnica de De-

\section{ETAPA 1: PROBLEMATIZAR O DEFINIR}

¿Cuáles fueron los principales asuntos de cada línea estratégica?
¿Cuáles fueron los principales problemas, limitaciones o barreras del asunto respectivo, durante la crisis sanitaria?
¿Cuál fue el grupo de interés mayormente impactado?
¿Que se aprendió durante la crisis sanitaria que permitiera potenciar el desempeño futuro de las operaciones? 


\section{ETAPA 2: PROPONER O IDEAR}

\begin{tabular}{|c|c|c|c|c|c|}
\hline \multicolumn{3}{|c|}{ La operación o actividad debió: } & \multirow{2}{*}{$\begin{array}{c}\text { Propuesta } \\
\text { ¿Qué iniciativas se } \\
\text { propusieron a partir de } \\
\text { las lecciones aprendidas, } \\
\text { el dialogo con terceros u } \\
\text { otras fuentes } \\
\text { referenciadas? }\end{array}$} & \multirow{2}{*}{$\begin{array}{c}\text { Beneficios } \\
\text { ¿Qué beneficios se esperaron } \\
\text { con las propuestas planteadas, } \\
\text { en términos de eficiencia, } \\
\text { oportunidad, seguridad, } \\
\text { competitividad, creación de } \\
\text { valor, preservación de valor u } \\
\text { otro? }\end{array}$} & \multirow{2}{*}{$\begin{array}{c}\text { Priorización } \\
\text { ¿Cuáles fueron } \\
\text { las propuestas } \\
\text { que se } \\
\text { consideraron } \\
\text { prioritarias? }\end{array}$} \\
\hline $\begin{array}{c}\text { ¿Seguir } \\
\text { operando como } \\
\text { antes de la } \\
\text { crisis? }\end{array}$ & $\begin{array}{c}\text { ¿Tener } \\
\text { ajustes } \\
\text { menores? }\end{array}$ & $\begin{array}{l}\text { ¿Tener } \\
\text { ajustes } \\
\text { mayores? } \\
\quad \text { (Ej. } \\
\text { Rediseño) }\end{array}$ & & & \\
\hline
\end{tabular}

\section{ETAPA 3: PROTOTIPAR - Solo para propuestas validadas y aprobadas}

Consiste en elaborar el diseño preliminar de la propuesta.

Pudo ser un esquema, un mapa conceptual, un flujo de operaciones, otro, que graficara o describiera en forma clara los elementos de entrada, las principales actividades o estrategias, sus interacciones, principales resultados e indicadores de evaluación.

Figura 4. Esquema diagramático de las etapas de Design Thinking para la formulación de las propuestas de la primera y segunda ronda.

Fuente: Tomado de Acuacar (2020)

No obstante, dada la oportunidad de aplicar al escrutinio un enfoque positivista en el marco de una mirada y diseño metódico transversal, se aplicó una encuesta $a d$ hoc a 185 de 453 funcionarios de Acuacar cuyos resultados permitieron contrastar las hipotesis aludidas a través de ecuaciones estructurales.

\section{Resultados}

Producto del método y técnica acotada en la tabla 2 se relacionan las propuestas iniciales (223) para restituir las operaciones paralizadas, después las palancas de mejora conclusivas (112) y al final lo que no quiere decir que no sean importantes, las susceptibles de prototipar (8). Cada una de las iniciativas resultó de un problema o constricción en los procesos y operaciones de Acuacar en deferencia a la pandemia Covid-19 en el marco de las 10 líneas estratégicas formuladas, pero en especial para garantizar la continuidad del negocio, las que luego de 2 rondas de validación y evaluación, se agruparon por afinidad procesal y línea estratégica en 115 propuestas de las cuales se descartaron 3 para un total de 112 .

Tabla 2. Propuestas por líneas estratégicas

\begin{tabular}{llccc}
\hline No & \multicolumn{1}{c}{ Lineas Estrategicas } & $\begin{array}{c}\text { No de Propuestas } \\
\text { Iniciales }\end{array}$ & $\begin{array}{c}\text { No de Propuestas } \\
\text { Finales }\end{array}$ & $\begin{array}{c}\text { No de Propuestas } \\
\text { para Prototipar }\end{array}$ \\
\hline 1 & Gestión de clientes & 45 & 17 & 2 \\
2 & Operaciones & 36 & 27 & 5 \\
3 & Organización del trabajo & 8 & 5 & 0 \\
4 & Regulación & 22 & 12 & 0 \\
5 & Gestión financiera & 22 & 11 & 0 \\
6 & Gestión ambiental & 12 & 8 & 1 \\
7 & Gestión social & 23 & 13 & 0 \\
8 & Seguridad y salud en el trabajo & 24 & 6 & 0 \\
9 & Relacionamiento con grupos de interés & 20 & 11 & 0 \\
10 & Gestión de Proyectos e Innovación & 11 & 2 & 0 \\
\hline \multicolumn{2}{r}{ Total } & $\mathbf{2 2 3}$ & $\mathbf{1 1 2}$ & $\mathbf{8}$ \\
\hline
\end{tabular}

Fuente: Tomado de Acuacar (2020) 
Como respuesta a la prototipación, se consideraron 8 iniciativas de mejoras aptas para pruebas piloto y de laboratorio para la construcción previa de unidades mínimas viables antes de escalar como soluciones definitivas, ver tabla 3. La mayoría circunscriben automatización y robotización de válvulas y componentes técnicos de los procesos de Captación, Tratamiento y Distribu- ción de agua, al igual que la digitalización de la información y procesos afines a la interpretación y toma de decisiones, así como nuevos modelos tecnológicos para el soporte de las rutinas humanas, en especial, los procesos inteligentes (Smart Processes por su nombre en inglés) basados en inteligencia artificial en lo que respecta a Facturación y Gestión de Cobro.

Tabla 3. Propuestas afines a prototiparse mediante unidades mínimas viables

\begin{tabular}{|c|c|c|}
\hline No & Líneas Estratégicas & Propuestas a prototipar \\
\hline 1 & Gestión de clientes & $\begin{array}{l}\text { Implementar el mecanismo para posibilitar la opción de que los usuarios a tra- } \\
\text { vés de la APP puedan suministrar la lectura y su respectiva evidencia fotográ- } \\
\text { fica a fin de evitar la presencia del lector en el predio, pero garantizando la } \\
\text { fiabilidad de la información. }\end{array}$ \\
\hline 2 & Gestión de clientes & $\begin{array}{l}\text { Incorporar la inteligencia artificial a la gestión de cartera que facilite el análisis } \\
\text { de datos de forma predictiva para direccionar las diferentes acciones de forma } \\
\text { asertiva y dinámica. }\end{array}$ \\
\hline 3 & Operaciones & $\begin{array}{l}\text { Automatización y robotización de rejas de acceso de las estaciones de Conejos, } \\
\text { Dolores y Gambote }\end{array}$ \\
\hline 4 & Operaciones & $\begin{array}{l}\text { Automatización y robotización de la dosificación de productos químicos, la lim- } \\
\text { pieza y drenaje de sedimentadores, el lavado de filtros del proceso tratamiento }\end{array}$ \\
\hline 5 & Operaciones & $\begin{array}{l}\text { Instrumentación y digitalización de las redes de acueducto por medio de una red } \\
\text { sensorial inteligente para el monitoreo en línea. }\end{array}$ \\
\hline 6 & Operaciones & $\begin{array}{l}\text { Automatización y robotización de sistemas de bombeo y subestaciones eléctri- } \\
\text { cas de agua cruda de la estación Dolores, Gambote, Piedrecitas y Conejos }\end{array}$ \\
\hline 7 & Operaciones & $\begin{array}{l}\text { Instrumentación y digitalización de las redes de acueducto a traves de movili- } \\
\text { dad del monitoreo en línea alarmas }\end{array}$ \\
\hline 8 & Gestión ambiental & Transformación digital para la gestión proactiva de olores \\
\hline
\end{tabular}

En la tabla 4, los criterios y reactivos para prototipar las propuestas en la ronda 3. Aquellas con evaluaciones superiores a 2 en los ítems de Complejidad, Duración de la Ejecución, Re- cursos Estimados y Redituabilidad consintieron exámenes experimentales previos. Las demás iniciativas (104) fueron aprobadas por el Comité de Resiliencia.

Tabla 4. Criterios para prototipar las propuestas

\begin{tabular}{|c|c|c|c|}
\hline \multirow{3}{*}{$\begin{array}{c}\begin{array}{c}\text { Criterios para } \\
\text { prototipar }\end{array} \\
\text { Complejidad }\end{array}$} & \multicolumn{3}{|c|}{ Escala de valoración para prototipar - unidades minimas viables } \\
\hline & \multirow{2}{*}{$\begin{array}{l}\text { Reactivo (1) } \\
\text { Actividades sencillas y fáci- } \\
\text { les de operar }\end{array}$} & Reactivo (2) & Reactivo (3) \\
\hline & & $\begin{array}{l}\text { Actividades básicas } \\
\text { con algún nivel de de- } \\
\text { talle }\end{array}$ & $\begin{array}{l}\text { Actividades detalladas y } \\
\text { competencias especializa- } \\
\text { das }\end{array}$ \\
\hline Duración & & Entre 3 y 6 Meses & Mas de 6 meses \\
\hline $\begin{array}{l}\text { Requerimiento de recur- } \\
\text { sos estimado }\end{array}$ & $\begin{array}{l}\text { Menor de } \$ 5 \text { Millones de pe- } \\
\text { sos colombianos o se encuen- } \\
\text { tra dentro del presupuesto }\end{array}$ & $\begin{array}{l}\text { Entre } 6 \text { y } \$ 20 \text { Millones } \\
\text { de pesos colombianos. }\end{array}$ & $\begin{array}{l}\text { Mayor de } \$ 20 \text { Millones } \\
\text { de pesos colombianos }\end{array}$ \\
\hline $\begin{array}{l}\text { Rentabilidad o retorno de } \\
\text { inversión }\end{array}$ & $\begin{array}{l}\text { Los beneficios son tangibles } \\
\text { y se obtienen antes de } 3 \text { me- } \\
\text { ses }\end{array}$ & $\begin{array}{l}\text { Los beneficios son tan- } \\
\text { gibles y se obtienen en- } \\
\text { tre } 3 \text { y } 6 \text { meses }\end{array}$ & $\begin{array}{l}\text { Los beneficios son tangi- } \\
\text { bles y se obtienen en tiem- } \\
\text { po superior a } 6 \text { meses }\end{array}$ \\
\hline
\end{tabular}

Fuente: Tomado de Acuacar (2020) 
Seguidamente en la tabla 5 los factores que posibilitaron las derivaciones de la corriente positivista para complementar el estudio.

Tabla 5. La lista preliminar de factores que afectan la resiliencia empresarial

\begin{tabular}{ll}
\hline \multicolumn{1}{c}{ Codigo } & \multicolumn{1}{c}{ Factores } \\
\hline CREA & $\begin{array}{l}\text { Creatividad } \\
\text { Flexibilidad para ser creativos en la pan- } \\
\text { demia y ser resilientes }\end{array}$ \\
CREA_1 & $\begin{array}{l}\text { Fluidez creativa en medio de la tensión } \\
\text { para ser resilientes }\end{array}$ \\
Integración digital y manejo óptimo de \\
datos
\end{tabular}

Fuente: Tomado de mediciones propias valoradas en Word (2020) con base en Acuacar (2020) y Arraut (2020)

El análisis de contraste en el cálculo del resultado de las variables independientes (creatividad, integración digital y manejo óptimo de datos, trabajo en equipo y análisis de la información) y la independiente (resiliencia), se realizó mediante SPSS y PLS, plataformas tecnológicas que posibilitan realizar investigaciones exploratorias.
Según Cohen (1998), el $f 2$ de las cuatro transformables observables sobre resiliencia arrojó una derivación pertinente sobre el valor de R2. E1 R2 dicho sea de paso fue del $75.81 \%$ lo que indica relevancia en la dependencia de las variables.

Tabla 6. Los efectos de variables independientes sobre la inconstante dependiente

\begin{tabular}{llc}
\hline Variables Latentes & Efectos f2 & \multicolumn{1}{c}{ Efecto total } \\
\hline Creatividad & 0.318 & Adecuado o pertinente \\
$\begin{array}{l}\text { Integración digital } \\
\text { y manejo optima de }\end{array}$ & 0.286 & \\
$\begin{array}{l}\text { datos } \\
\text { Trabajo en equipo }\end{array}$ & 0.278 & Adecuado o pertinente \\
$\begin{array}{l}\text { Analisis de la infor- } \\
\text { mación }\end{array}$ & 0.285 & Adecuado o pertinente \\
\hline
\end{tabular}

Fuente: Tomado de mediciones propias valoradas en SPSS y PLS (2020)

En el SEM o en el modelo de ecuaciones estructurales, los valores de Q2 medidos (resultantes del PLS) deben ser mayores que cero para una construcción latente endógena. En la Figura 5, se enseña que el valor de Q2 para este modelo fue de 0.438 , resultado mayor que el límite umbral, y en tal virtud, respaldó la naturaleza predictiva del modelo. 


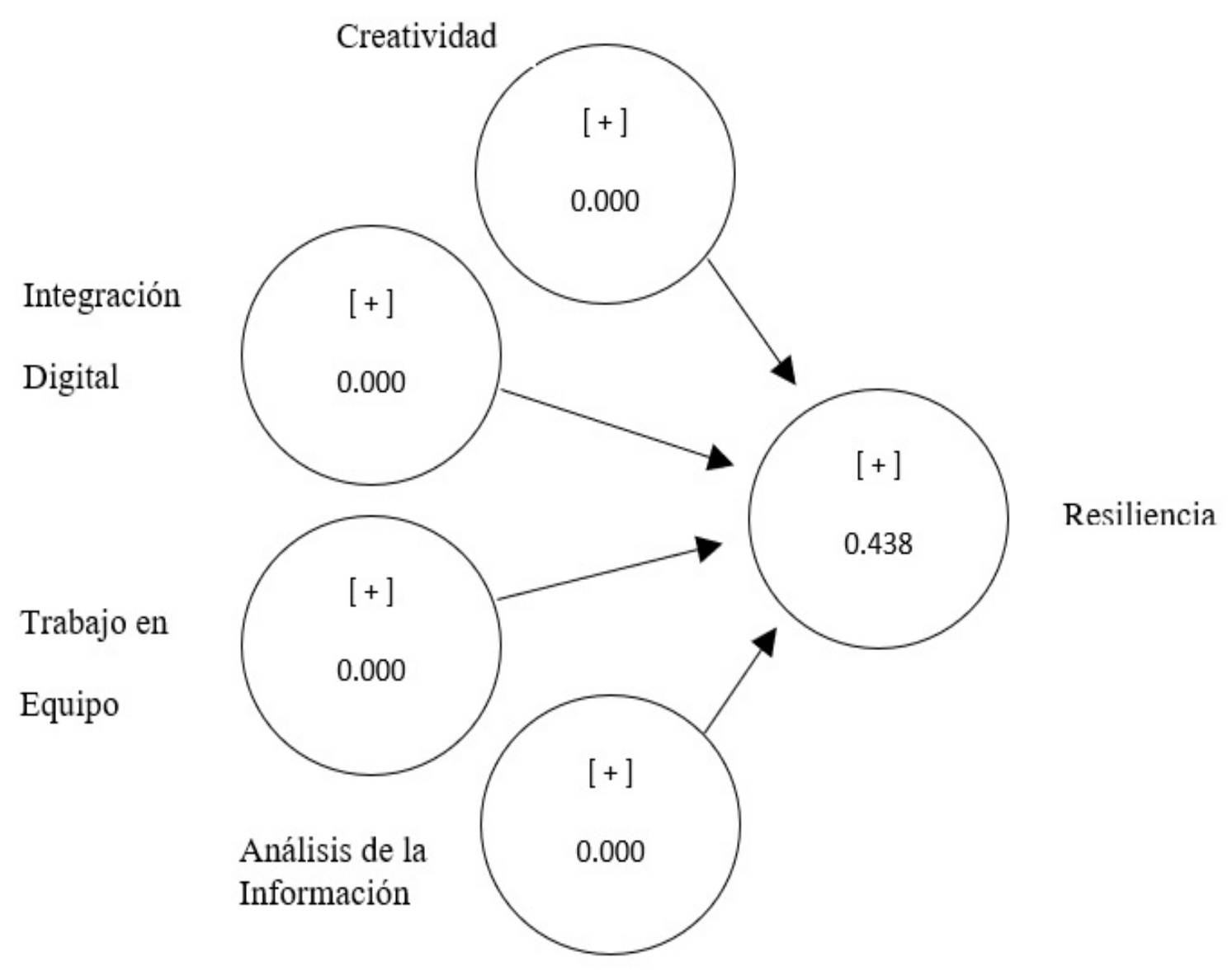

Figura 5. Modelo predictivo

Fuente: Preparación a partir de cálculos en SPSS y PLS (2020)

La métrica o índice de bondad de ajuste (GOF por su sigla en inglés) se aplicó al modelo a condición de verificar si explicase ampliamente los datos empíricos. Los valores GOF se encontraron entre 0 y 1 , donde los valores de 0.10 (pequeños), 0.25 (medianos) y 0.36 (grandes) indicaron la validación global del modelo.
El ajuste del modelo enseñó un patrón parsimonioso y plausible. El GOF finalmente, se calculó utilizando el valor medio geométrico de la comunalidad promedio o varianza media extraída (valores AVE por su sigla en inglés) y los valores promedio de R2.

Tabla 7. Cálculo del índice GOF

\begin{tabular}{ccc}
\hline Constructos & AVE & R2 \\
Creatividad & 0.658 & \\
Integración digital & 0.596 & \\
Trabajo en equipo & 0.652 & \\
Analisis de la informa- & 0.652 & 0.7399 \\
ción & 3.779 & 0.7399 \\
Average Values & 0.4949 & \\
AVE $*$ R2 & 0.7035 & \\
GOF $=\sqrt{ }$ AVE $*$ R2
\end{tabular}

Fuente: Tomado de mediciones propias valoradas en SPSS y PLS (2020)

El índice del promedio de residuos estandarizados (SRMR por su sigla en ingles) entre los cálculos realizados y las hipótesis de las matrices de covarianza fue de 0.043 , en tal condición se encuentra pertinente $(\mathrm{SRMR}=<0.08)$, en consecuencia; el modelo tiene buen ajuste. Por su parte, el Chi cuadrado fue igual a 1914.085 y el índice normado de ajuste (NFI por sus siglas en inglés) arrojó 0.789 por lo que también se considera adecuado el patrón de medida. 
Tabla 8. Estimadores del modelo

\begin{tabular}{ll}
\hline & Estimadores del Modelo \\
\hline SRMR & 0.043 \\
d_ULS & 1.622 \\
d_G1 & 0.914 \\
d_G2 & 0.767 \\
Chi-Square & 1.914 .085 \\
NFI & 0.789 \\
\hline Tomado de mediciones propias valoradas en SPSS y PLS (2020)
\end{tabular}

Finalmente, la tabla 9 enseña los coeficientes entre las variables exógenas latentes y las obserde correlación de las variables latentes, la que vables endógenas. permite inferir que hubo una fuerte correlación

Tabla 9. Correlación de las variables latentes y observables

\begin{tabular}{lcccc}
\hline & CREA & INT & APR & ANA \\
Creatividad & $\mathbf{1 . 0 0 0}$ & & & \\
Integración digital & 0.245 & $\mathbf{1 . 0 0 0}$ & & \\
Trabajo en equipo & 0.268 & 0.255 & $\mathbf{1 . 0 0 0}$ & \\
Analisis de la información & 0.256 & 0.297 & 0.268 & $\mathbf{1 . 0 0 0}$ \\
\hline \multicolumn{4}{c}{ Fuente: Tomado de mediciones propias valoradas en SPSS y PLS (2020) }
\end{tabular}

Con base en el análisis del modelo de medición, se estableció su viabilidad confirmatoria. Todas las conjeturas en tal virtud fueron estadísticamente significativas, por lo que se aceptan. Las derivaciones de esta investigación respaldan que los factores acotados afectaron positivamente la resiliencia por lo que podrían apoyar la generalidad literaria en torno a dicho constructo obviamente sí los ejercicios que realicen otros investigadores se cometan bajo los mismos lineamientos.

\section{Conclusión y discusión}

En conclusión, el trabajo de investigación respondió la pregunta central: ¿cómo robustecer la resiliencia organizacional en la empresa Aguas de Cartagena de tal suerte que le permitiera operar normalmente en tiempos Covid-19? Al respecto conviene decir que el método adoptado admitió fortalecer la capacidad de recuperación de las operaciones de la empresa que se realizan con contratistas $(51 \mathrm{de}$ 112 operaciones, 61 las realiza ACUACAR con su personal directo) ante la pandemia puesto que del $50 \%$ de los procesos suspendidos donde participan proveedores de servicios en la actualidad solo continúan paralizados el 3\%, lo anterior en respuesta a las 115 de 223 propuestas entregadas por los equipos de trabajo que conformaron el ejercicio práctico. De ellas, 8 son susceptibles de prototiparse por medio de unidades experimentales y 3 fueron descartadas para un total de 112. Es importante acotar de igual manera que, el restablecimiento de las operaciones obstaculizadas demanda autorizaciones del gobierno nacional colombiano.

La investigación también posibilitó concluir que la gestión de resiliencia en la empresa Aguas de Cartagena implicó el involucramiento tanto de los procesos neurálgicos que fueron detenidos a raíz de la pandemia como de los colaboradores de la firma, incluyendo personas internas y ajenas a estas rutinas porque en el trabajo participaron individuos externos (miembros de empresas externas) e internos conformantes de 10 grupos focales afines a las 10 líneas estratégicas indicadas. Todo esto confirma la posición epistemológica de la resiliencia empresarial holística mencionada en la fundamentación teórica, respaldando las tesis de Hamel y Välikangas (2003), Hoffman (2014), McManus, Seville, Brunsdon y Vargo (2007) y Prezelj y Doerfel (2017), pero contrariando el punto de vista de Meneghel, Salanova y Martínez (2013), Salanova, Llorens, Cifre y Martínez (2012) y Somsing y Belbaly (2017), quienes las consideran antropomorfizadas e individuales y grupales. 
Por su parte, en el paradigma positivista, los factores: creatividad, integración digital, trabajo en equipo y análisis de la información numérica afectaron positivamente la resiliencia empresarial respaldando la posición de (Arraut, comunicación personal, 23 y 28 de agosto de 2020) y Somsing y Belbaly (2017).

\section{Limitaciones y futuras Investigaciones}

La limitante más importante es que el análisis no incluyó los contratistas de servicios mucho menos los proveedores de insumos, los que cuentan con unos 800 empleados, incluyendo sus equipos directivos. En el futuro también podrían incluirse estas organizaciones.

\section{Referencias}

Cohen, J. (1988). Statistical Power Analysis for the Behavioral Sciences. NJ Lawrence Earlbaum Assoc, 2, 567.

Doerfel, M. L. (2016). Networked forms of organizing, disaster-related disruptions, and public health. In T. R. Harrison \& E. A. Williams (Eds.). Organizations, communication, and health, 365-383. New York, NY: Routledge.

García-Merino, T., Rivera, H.A. y Santos-Álvarez, V. (2015). La formación del concepto de resiliencia empresarial: Análisis cualitativo para el caso de los/as percebeiros/as de la cofradía de Baiona (España). Forum: Qualitative Social Research Sozial Forschung, 16(3), Art. 34, http://nbn-resolving.de/urn:nbn:de:0114-fqs1503349.

Hamel, G. y Välikangas, L. (2003). En busca de la resiliencia. Harvard Business Review, 1(9).

Hoffman, H. (2014). La resiliencia como estrategia organizacional. In Forum Humanes, 2(2), 67-75.

Hollnagel, E. (2006). Resilience Engneering: Concepts and precepts. Reino Unido: Ashgate. Luthar, S. S., Cicchetti, D. y Becker, B. (2000). The construct of resilience: a critical evaluation and guidelines for future work. Child Development, 71(3), 543-562.

McManus, S., E. Seville, D. Brunsdon y J. Vargo (2007). Resilience management. A framework for assessing and improving the resilience of organizations. Reporte de investigación 07/1, Nueva Zelanda: Universidad de Canterbury.

Medina-Salgado, C. (2012). La resiliencia y su empleo en las organizaciones. Harvard Business Review, 3(19).

Meneghel, I., Salanova, M., y Martínez, I. M. (2013). El camino de la Resiliencia OrganizacionalUna revisión teórica. Aloma: Revista de Psicologia, Ciències de l'Educació i de l'Esport, $31(2)$.

Mendoza, J. M. (2013). La capacidad dinámica de ripostar en la empresa: Confrontar entornos volátiles. Cuadernos de Administración, 26(47), 63-85.

Mendoza-Betin, J. A. (2018). Capacidades dinámicas: Un análisis empírico de su naturaleza. Revista MLS Educational Research (MLSER), 2(2), 76-92. ISSN 2603-5820.

https://www.mlsjournals.com/Educational-Research-Journal/article/view/80/185.

Mendoza-Betin, J. A. (2019). Capacidades Dinámicas y Rentabilidad Financiera: Análisis desde una perspectiva ecléctica en empresas de Saneamiento Básico de Cartagena. Tesis doctoral. Universidad Internacional Iberoamerica, Mexico.

Minolli, C. B. (2005). Empresas resilientes algunas ideas para construirlas. Temas de Management, $3(1), 20-25$. 
Prezelj, I. (2014). Inter-organizational cooperation and coordination in the fight against terrorism: From undisputable necessity to paradoxical challenges. Comparative Strategy, 33(4), 321341.

Prezelj, I. y Doerfel, M. (2017). Resilience in a complex and unpredictable world. J Contingencies and Crisis Management, 25, 118-122.

Salanova, M., Llorens, S., Cifre, E., \& Martínez, I. M. (2012). We need a hero! Toward a validation of the healthy and resilient organization (HERO) model. Group \& Organization Management, $37(6), 785-822$.

Somsing, A. y Belbaly, N.A. (2017). Managerial Creativity: The Roles of Dynamic Capabilities and Risk Preferences. European Management Review, 14, 423-437.

Sutcliffe, K. y T. Vogus (2007). The safety organizing scale: Development and validation of a behavioral measure of safety culture in hospital nursing units. Medical Care, 45(1), 46-54. 